\title{
Progress in the Application of Carbon Dots-Based Nanozymes
}

\author{
Jun Jin, Linlin Li, Lihui Zhang, Zhihui Luan, Shuquan Xin * and Kai Song * \\ School of Life Sciences, Changchun Normal University, Changchun, China
}

As functional nanomaterials with simulating enzyme-like properties, nanozymes can not only overcome the inherent limitations of natural enzymes in terms of stability and preparation cost but also possess design, versatility, maneuverability, and applicability of nanomaterials. Therefore, they can be combined with other materials to form composite nanomaterials with superior performance, which has garnered considerable attention. Carbon dots (CDs) are an ideal choice for these composite materials due to their unique physical and chemical properties, such as excellent water dispersion, stable chemical inertness, high photobleaching resistance, and superior surface engineering. With the continuous emergence of various CDs-based nanozymes, it is vital to thoroughly understand their working principle, performance evaluation, and application scope.

Edited by:

Paul E.D. Soto Rodriguez, Commissariat à l'Energie Atomique et aux Energies Alternatives (CEA), France

Reviewed by: Maria Guix,

Institute for Bioengineering of Catalonia (IBEC), Spain Jiabin Cui,

Soochow University, China

${ }^{*}$ Correspondence: Kai Song songkai@ccsfu.edu.cn Shuquan Xin xinshuquan@qq.com

Specialty section:

This article was submitted to Nanoscience,

a section of the journal Frontiers in Chemistry

Received: 27 July 2021 Accepted: 10 September 2021 Published: 24 September 2021

Citation:

Jin J, Li L, Zhang L, Luan Z, Xin S and Song $K$ (2021) Progress in the Application of Carbon DotsBased Nanozymes.

Front. Chem. 9:748044. doi: 10.3389/fchem.2021.748044
This review comprehensively discusses the recent advantages and disadvantages of CDs-based nanozymes in biomedicine, catalysis, sensing, detection aspects. It is expected to provide valuable insights into developing novel CDs-based nanozymes.

Keywords: nanozymes, carbon dots, biomedicine, catalysis, sensing, detection

\section{INTRODUCTION}

Natural proteases are easily denatured and degraded under harsh environmental conditions, their catalytic efficiency is limited, and their product separation and purification are costly. Their recovery and recycling are difficult, dramatically limit their practical applications (Attar et al., 2019; Wang Z. et al., 2020; Ding et al., 2020). For instance, although considerable progress has been made in the design and development of catalytic nanomotors such as bimetallic nanorods, catalytic microtubes, Janus particles and bioenzyme-driven motors, some problems remain, such as a small number of applied enzymes, a slow motor speed, and toxicity of high hydrogen oxide $\left(\mathrm{H}_{2} \mathrm{O}_{2}\right)$ concentrations (Ma et al., 2016; Xu et al., 2019; Hermanova and Pumera, 2020; Hermanova and Pumera, 2020; Mathesh et al., 2020; Yang Q. et al., 2021; Yuan et al., 2021).

In this case, it is necessary to identify a suitable enzyme substitute to simulate the natural enzyme. Since Yan and his colleagues first demonstrated the peroxidase activity of magnetic $\mathrm{Fe}_{3} \mathrm{O}_{4}$ nanoparticles (NPs) in 2007, numerous nanomaterials mimicking enzymes have been developed (Gao et al., 2007; Natalio et al., 2012; Hou et al., 2013; Wei and Wang, 2013; Lin et al., 2014; Kluenker et al., 2017). In addition, the researchers are exploring ways to integrate other nanomaterials with nanozymes to improve the catalytic efficiency of cascade reactions. For example, integrated nanozyme invertase/GOx/hemin@ZIF-8A has a 700\% higher catalytic efficiency than mixed invertase@ZIF-8, GOx@ZIF-8, and hemin@ZIF-8 alone (Cheng et al., 2016).

CDs are excellent candidates for nanomaterial composites with nanozymes due to their surface modification, heteroatom doping, and composite with NPs (Kang and lee., 2019; Yang et al., 2020; Wang et al., 2019). In recent years, although CDs-based nanozymes have successfully simulated the structure and function of common natural enzymes such as oxidase, catalase and superoxide 


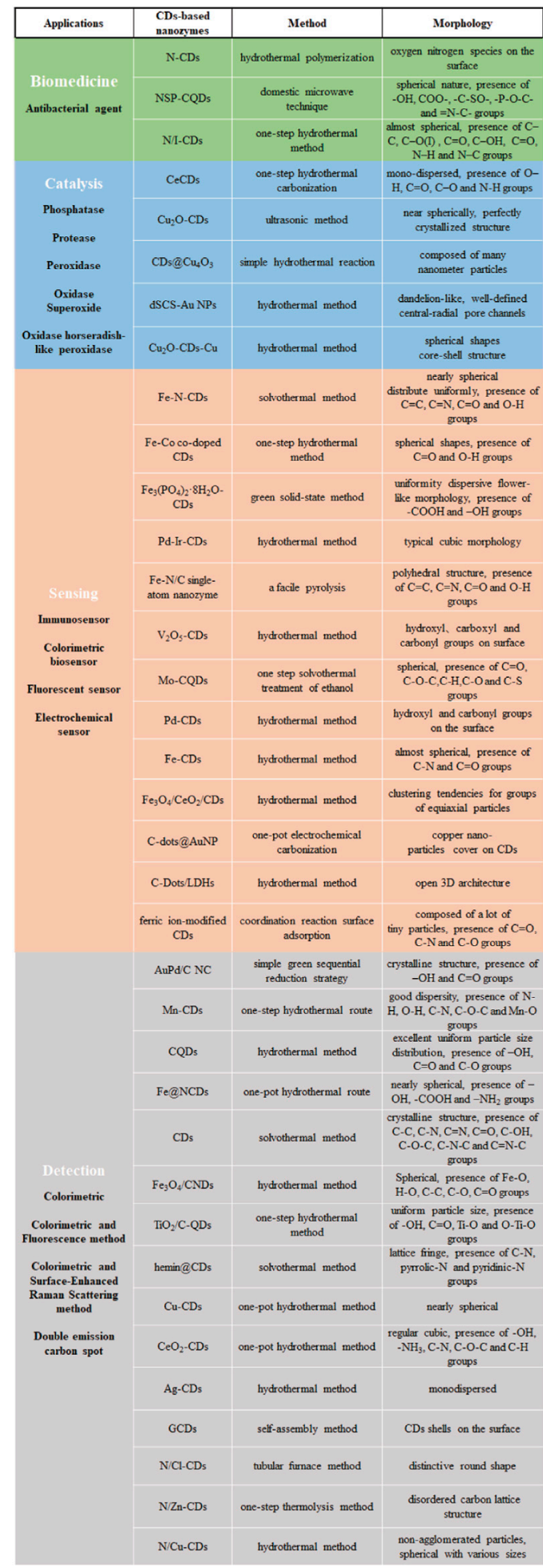

FIGURE 1 | The synthesis method and structural property of CDs-based nanozymes.

dismutase, they continue to face numerous obstacles (Zhao et al., 2020; Li et al., 2020a). The most significant limitation is that catalytic reactions are relatively few in number, with a strong emphasis on reduction-oxidation (REDOX) reactions. As a result, it is necessary to summarize the application research of CDsbased nanozymes with different sources and structural characteristics (Figure 1), which can provide a reference for future searching or designing novel nanozymes.

\section{THE APPLICATIONS OF CDs-BASED NANOZYMES IN BIOMEDICINE}

Biomedicine urgently requires the development of effective antimicrobial agents to combat bacterial contamination. Although antibiotics, metal NPs, composite NPs, and enzymes have been employed as antimicrobial agents, these materials exhibit several limitations: cytotoxicity, antibiotic resistance, and environmental pollution (Fischbach and Walsh, 2009; Kohanski et al., 2010; Song et al., 2012; Fasciani et al., 2014; Rizzello and Pompa, 2014; Leidinger et al., 2015). Therefore, there is a great demand for low-cost, sustainable, and effective antimicrobials suitable for long-term use. CDs-based nanozymes are an effective alternative to the above materials due to their unique electronic, optical, thermal, and mechanical properties. Zhang et al. synthesized a series of nitrogen-doped CDs to mimic the activity of oxidase. Such CDs can mimic the oxidation reaction in a few seconds and effectively inhibit the growth of Escherichia coli (E. coli) and Salmonella (Zhang et al., 2018). However, it demonstrated antibacterial activity only at acidic $\mathrm{pH}$ and insufficient activity at physiological conditions around neutral $\mathrm{pH}$. For this reason, Kumud Malika Tripathi et al. prepared luminescent N, S, and P-co-doped carbon quantum dots (NSPCQDs) that exhibited peroxidase activity over a wide $\mathrm{pH}$ range attributed to the presence of a high density of active sites for enzymatic-like catalysis and accelerated electron transfer during peroxidase-like reactions. It can significantly inhibit cell wall growth of E. coli and Staphylococcus aureus (Tripathi et al., 2020). Although this study realized the antibacterial effect of CDs-based nanozymes, it did not consider the toxicity issues associated with a high $\mathrm{H}_{2} \mathrm{O}_{2}$ concentration. Therefore, Wang et al. used a hydrothermal method to synthesize a novel nitrogen-iodine co-doped CDs (N/ I-CDs) with excellent peroxidase activity. When activated by light, they catalyze the conversion of exogenous $\mathrm{H}_{2} \mathrm{O}_{2}$ into hydroxyl radical $(\mathrm{OH})$, reduce high concentration of $\mathrm{H}_{2} \mathrm{O}_{2}$ to benign biological concentration $(50-100 \mu \mathrm{M})$, and increase the cell level of reactive oxygen species (ROS) in bacterial cells. They also effectively resist Gram-negative and Gram-positive bacterial infection and accelerate the healing of artificial wounds (Wang X. et al., 2021).

At present, only a few reports are evaluating the antibacterial properties of CDs-based nanozymes. In addition, whether CDsbased nanozymes can inhibit fungi or viruses is a field worthy of research (Fan et al., 2018).

\section{THE APPLICATIONS OF CDs-BASED NANOZYMES IN CATALYSIS}

Most catalytic reactions of nanozymes are mainly focused on peroxidase, oxidase, superoxide oxidase, and catalase reactions, while natural enzymes are diverse and exhibit various catalytic 
capabilities, developing nanozymes for new enzyme reactions is highly demanding (Gao et al., 2007; Asati et al., 2009; Wei and Wang, 2013; Lin et al., 2014). For instance, Ce-doped CDs (CeCDs) can simulate phosphatase activity, which is used for phosphate ester hydrolysis (Du et al., 2020). However, the optimal reaction conditions for this nanozyme are an alkaline solution with $\mathrm{pH} 8.5$ and a high temperature of $200^{\circ} \mathrm{C}$. These harsh reaction conditions significantly limit its application in biological systems. $\mathrm{Li}$ et al. attempted to synthesize $\mathrm{Cu}_{2} \mathrm{O}$-decorated carbon quantum dots $\left(\mathrm{Cu}_{2} \mathrm{O}\right.$-CDs) with intrinsic protease-simulating activity, which hydrolyzed proteins including bovine serum albumin and casein under physiological conditions (Li et al., 2020a). This dramatically improves the applicability of nanozymes in proteomics and related fields, opening the door to a plethora of potential biological applications.

As many biochemical processes are carried out by various enzymes, studying nanozymes simulating complex enzyme reactions is one of the demanding research goals. $\mathrm{Li}$ et al. studied paramelaconite $\left(\mathrm{CDs} @ \mathrm{Cu}_{4} \mathrm{O}_{3}\right)$ with both oxidase and peroxidase activities ( $\mathrm{Li}$ et al., 2018). Zhao et al. synthesized dual nanozymes with a complex CDs, which realized the simultaneous dual catalysis of superoxide dismutase and horseradish peroxidase activities (Zhao et al., 2020). These CDs-based nanozymes provide a new perspective on synergistic properties and comprehensive functions beyond traditional nanozymes. In addition, the properties of composite materials can confer the nanozymes new properties, such as stimulus responsiveness. $\mathrm{Li}$ et al. synthesized $\mathrm{Cu}_{2} \mathrm{O}$-CDs- $\mathrm{Cu}$ three component oxidase-like catalyst, which can effectively generate high-energy electrons under visible light irradiation to improve its oxidase catalytic activity (Li et al., 2020b). This study provides insights into the design of catalysts that can effectively couple thermal and photonic stimuli to drive oxidase-like activity.

The catalytic mechanism of CDs-based nanozymes is not fully understood. Although the active intermediates, catalytic activity, and substrate binding sites have been identified, the progression of reactions remains unclear.

\section{THE APPLICATIONS OF CDS-BASED NANOZYMES IN SENSING}

As an ideal and essential tool of biosensors, nanozymes have attracted great attention because of their lower cost, higher stability and more convenient preparation than protein enzymes. Inorganic nanomaterials with various enzymatic activities, such as ferromagnetic NPs, AuNP@MoS 2 QD gold NPs, and $\mathrm{MoS}_{2}$ Nanoribbons, have been explored as biosensors (Wei and Wang, 2013; Woo et al., 2013; Nirala et al., 2015; Liu et al., 2018; Vinita et al., 2018; Ding et al., 2019).

\section{The Immunosensor}

Yang et al. synthesized iron and nitrogen co-doped CDs (Fe-NCDs), which with peroxidase activity. 3,3',5,5'tetramethylbenzidine (TMB) was catalyzed to blue in the presence of hydrogen peroxide. On this basis, Fe-N-CDs conjugated antibody was applied to detect carcinoembryonic antigen (CEA) by immunosorbent assay. The detection limit was as low as $0.1 \mathrm{p} \mathrm{g} / \mathrm{mL}$ within $5 \mathrm{~min}$ (Yang et al., 2017). Based on the similar principle of enzyme-linked immunosorbent assay, iron and cobalt co-doped CDs with high peroxidase-like activity and palladium-iridium nanocubes with $\mathrm{CDs}$ as reference fluorophores can detect histamine and cardiac troponin I, respectively (Tan et al., 2019; Li et al., 2021). Even more striking, Guo et al. used $\mathrm{Fe}_{3}\left(\mathrm{PO}_{4}\right)_{2} \cdot 8 \mathrm{H}_{2} \mathrm{O}$-CDs-FA hybrid nanoflower realized the naked eye immunoassay of as few as $25 \mathrm{HeLa}$ cells (Guo et al., 2019).

\section{The Colorimetric Biosensor}

Based on the above TMB discoloration principle, Fe-N/C singleatom nanozyme was used to screen alkaline phosphatase activity in the range of $0.05-100 \mathrm{U} / \mathrm{L}$, with a detection limit of $0.02 \mathrm{U} / \mathrm{L}$ (Chen Q. et al., 2020). The cascade colorimetric biosensor combined with cholesterol oxidase demonstrated excellent selectivity and high sensitivity to the target in the concentration range of $0.01-1.0 \mathrm{mM}$. The detection limit was as low as $7 \mathrm{mM}$ (Zhao et al., 2019). Both $\mathrm{V}_{2} \mathrm{O}_{5}-\mathrm{CDs}$ nanocomposites and palladium/CDs composites (Pd-CDs) have also been proved to bind glucose oxidase and realize the colorimetric glucose sensing with a detection limit as low as $0.2 \mu \mathrm{M}$ (Honarasa et al., 2019).

\section{The Fluorescent Sensors}

CDs have demonstrated significant application value in fluorescence detection due to their numerous unique physical and photochemical properties, and CDs-based nanozymes also exhibit fluorescence detection characteristics (Zhan et al., 2020).

Lu et al. synthesized Fe-doped CDs (Fe-CDs). Oxidative OPD (ox-OPD) can be generated when the oxidase substrate o-phenylenediamine (OPD) coexists with $\mathrm{H}_{2} \mathrm{O}_{2}$. Therefore, a dual fluorescence emission detection system can be established based on fluorescence characteristics of Fe-CDs and Ox-OPD. The results indicated that the limit of detection for cysteine was as low as $0.047 \mu \mathrm{M}$ in the concentration range of $0.25-90 \mu \mathrm{M}(\mathrm{Lu}$ et al., 2020).

\section{The Electrochemical Sensors}

The advantages of electrochemical sensors include linear output, low power consumption, good resolution, repeatability, and accuracy (Chen et al., 2019; Teymourian et al., 2020; Wang Q. et al., 2021). Additionally, applying CDs-based nanozymes to electrochemical sensors is a hot topic.

The realization of electrochemical sensing based on CDsbased nanozymes is often the modification of electrodes. Wang et al. immobilized horseradish peroxidase on a glassy carbon electrode by simply mixing carbon nanodots and cobalt-iron layered double hydroxides (Wang et al., 2015). Qin et al. used hydroxyl-rich carbon dot-assisted gold nanoparticles (CDs @AuNP) as a marker of copper deposition reaction, and cooperated with chitosan to modify glassy carbon electrode (Qin et al., 2018). Hu et al. used coordination reaction and surface adsorption to prepare ferrous and ferrous ion modified CDs to regulate heterogeneous nucleation process of iron 
oxide, and its enzyme-like activity was more than 6 times higher than that of pure $\mathrm{Fe}_{2} \mathrm{O}_{3}$ nanomaterials (Hu et al., 2021) Fatemeh Honarasa et al. prepared $\mathrm{Fe}_{3} \mathrm{O}_{4} / \mathrm{CeO}_{2} / \mathrm{C}$-dot nanozyme with more complex structure, and its modified multi-walled carbon nanotube/ionic liquid paste (MWIL) electrode was used for electrocatalytic determination of $\mathrm{H}_{2} \mathrm{O}_{2}$, showing a linear range of $2.0 \times 10^{-8} \sim 1.0 \times 10^{-6} \mathrm{M}$ (Honarasa et al., 2021).

Compared with metal/metal oxide NPs or materials, CDs have the disadvantages of low product yield, difficulties in purification and precise size control, which significantly affect applying CDsbased nanozymes in biosensors.

\section{THE APPLICATIONS OF CDS-BASED NANOZYMES IN DETECTION}

\section{The Colorimetric Detection}

Biomolecules such as $\mathrm{H}_{2} \mathrm{O}_{2}$, ascorbic acid, uric acid, and pyrophosphate have also been developed to detect the nanozyme complex CDs method.

Yang et al. synthesized carbon-based AuPd bimetallic nanocomposite (AuPd/C NC) with good catalytic activity and peroxidase activity. $\mathrm{H}_{2} \mathrm{O}_{2}$ can be detected in a wide linear concentration range of $5-500 \mu \mathrm{M}$ and $500 \mu \mathrm{M}-4 \mathrm{mM}$ (Yang et al., 2016). Zhuo et al. demonstrated that manganese (II) doped CDs (Mn-CDs) have a similar catalytic ability to oxidase. They could be utilized for quantifying ascorbic acid in a concentration range of 50-2,500 $\mathrm{nM}$ based on the principle of “TMB discoloration reaction" (Zhuo et al., 2019). Shu et al. demonstrated that carbon quantum dots (CQDs) also exhibit peroxidase activity but with a narrower detection range and lower detection limit (Shu et al., 2020). Liang et al. synthesized carbon quantum dots co-doped with iron and nitrogen (Fe@NCDs). In the presence of $\mathrm{H}_{2} \mathrm{O}_{2}$, the response was linear in the uric acid concentration range of $2-150 \mu \mathrm{M}$ (Liang et al., 2020). Chen et al. prepared nanozymes with complex CDs exhibiting peroxidase simulation properties, which could catalyze o-phenylenediamine oxidation in the presence of $\mathrm{H}_{2} \mathrm{O}_{2}$. The process was inhibited by pyrophosphate (PPI), and the degree to which it was inhibited could be monitored using the colorimetric method with generated yellow product 2,3-diaminophenazine (Chen Q. et al., 2020).

Although nanozyme-based colorimetry is a rapid method for detecting glutathione, it lacks the high efficiency and low toxicity of nanozyme. Luo et al. prepared $\mathrm{Fe}_{3} \mathrm{O}_{4} / \mathrm{CND}$ hybrid NPs with excellent peroxidase-like catalytic activity, and they could produce a rapid color reaction on glutathione (Luo et al., 2019). Similar studies have focused on peroxidase-like nanomaterials, which require $\mathrm{H}_{2} \mathrm{O}_{2}$ addition. Because $\mathrm{H}_{2} \mathrm{O}_{2}$ is extremely unstable, quickly decomposes, and even reacts with assay, applying this nanozyme mimicking peroxidase remains limited. Therefore, Jin et al. prepared titanium dioxide/carbon point oxidase nanozyme. The nanozyme possessed abundant thermodynamic metastable $\mathrm{Ti}$ atoms on MXene. The oxygen vacancy in $\mathrm{TiO}_{2}$ on carbon matrix surface can facilitate $\mathrm{O}_{2}$ adsorption in solution, generating ROS, thereby quickly oxidizing TMB to TMBox in the absence of $\mathrm{H}_{2} \mathrm{O}_{2}$ to detect glutathione (Jin et al., 2020).

\section{Collaborative Detection by Colorimetric Method and Fluorescence Method}

Although colorimetric and fluorescence methods possess high selectivity, high sensitivity, low cost, and simplicity, such methods follow single-mode signal readout. It is easy to be disturbed by the environment and challenging to meet accurate bioassay requirements. In this case, colorimetric/fluorescence twochannel measurement provides a more reliable strategy for detecting $\mathrm{H}_{2} \mathrm{O}_{2}$ and related biomolecules.

Su et al. prepared for the first time a multifunctional hemin@ CDs hybrid nanozymes (hemin@CDs) with peroxidase-like activity and fluorescence signal properties ( $\mathrm{Su}$ et al., 2020). This is a two-channel fluorescent probe for $\mathrm{H}_{2} \mathrm{O}_{2}$ and $\mathrm{H}_{2} \mathrm{O}_{2}$ based biocatalytic systems. It catalyzes the oxidative coupling of 4-aminoantipyrine and phenol in the presence of $\mathrm{H}_{2} \mathrm{O}_{2}$, resulting in a pink quinone imine dye with a maximum absorbance at $505 \mathrm{~nm}$. The probe can be deployed to detect glucose and xanthine due to the conversion of glucose/xanthine into $\mathrm{H}_{2} \mathrm{O}_{2}$ catalyzed by related oxidase.

Ren et al. synthesized active copper-containing CDs (Cu-CDs) with inherent laccase-like activity. Unlike Su et al.'s work, this is a novel enzyme reaction that catalyzes phenylenediamine oxidation by laccase substrates, resulting in a typical color change from colorless to brown. $\mathrm{Cu}$-CDs were further employed as a fluorescent probe for unlabeled hydroquinone $\left(\mathrm{H}_{2} \mathrm{Q}\right)$ detection. The results indicate that a linear relationship is good in buffers with different $\mathrm{pH}$ values of $0.05-20 \mathrm{mM}$ and 1-30 mM (Ren et al., 2015).

To further overcome the problem of obtaining fluorescence utterly dependent on a single signal output and a low signal background ratio in the method mentioned above, Yang et al. prepared CDs-doped $\mathrm{CeO}_{2}\left(\mathrm{CeO}_{2}\right.$-CDs) with peroxidase activity and fluorescent carbon dot. Fluorescent o-phenylenediamine (OPD), a peroxidase substrate, can be catalyzed by cerium oxide and cadmium sulfide to produce fluorescent o-phenylenediamine (palladium oxides). UV-Vis absorption of palladium oxides partially overlays the fluorescence emission of cadmium sulfide, reducing its intensity under the effect of an internal filter (Yang Z. et al., 2021). Based on this principle, a sensitive and selective fluorescence assay for the ratio of $\mathrm{H}_{2} \mathrm{O}_{2}$ to cholesterol was developed.

\section{Collaborative Detection by Colorimetric Method and Surface-Enhanced Raman Scattering (SERS) Method}

Gold and silver are typical SERS substrates. The SERS activity of precious metals/CDs nanocomposites was enhanced by improving probe molecule adsorption and amplifying electromagnetic fields.

Wang et al. prepared silver-CDs (Ag-CDs) nanocomposites with excellent peroxidase and SERS activity. The nanocomposite can be used to determine uric acid (UA) levels (Wang et al., 2019). In addition, the chain-like $\mathrm{Au} / \mathrm{CDs}$ (GCDs) nanocomposite was 
TABLE 1 | Summary of the application of CDs-based nanozymes in detection.

\begin{tabular}{|c|c|c|c|c|}
\hline Detection method & Sample & Linear range & Detection limit & References \\
\hline Colorimetric Detection & $\mathrm{H}_{2} \mathrm{O}_{2}$ & $\begin{array}{c}5-500 \mu \mathrm{M} \\
500 \mu \mathrm{M}-4 \mathrm{mM}\end{array}$ & $0.16 \mu \mathrm{M}$ & Yang et al. (2016) \\
\hline- & Glutathione & $0.058 \mu \mathrm{M}$ & - & Luo et al. (2019) \\
\hline- & - & $0.5-25 \mu \mathrm{M}$ & $0.2 \mu \mathrm{M}$ & Jin et al. (2020) \\
\hline- & Ascorbic acid & $50-2500 \mathrm{nM}$ & $9 \mathrm{nM}$ & Zhuo et al. (2019) \\
\hline- & - & $1.0-105 \mu \mathrm{M}$ & $0.14 \mu \mathrm{M}$ & Shu et al. (2020) \\
\hline- & Uric acid & $2-150 \mu \mathrm{M}$ & $0.64 \mu \mathrm{M}$ & Liang et al. (2020) \\
\hline- & $\begin{array}{l}\text { Pyrophosphate } \\
\text { lon }\end{array}$ & - & $4.29 \mathrm{nM}$ & Chen et al. (2020b) \\
\hline $\begin{array}{l}\text { Collaborative detection by colorimetric and fluorescence } \\
\text { methods }\end{array}$ & $\mathrm{H}_{2} \mathrm{O}_{2}$ & - & $\begin{array}{l}0.11 \mu \mathrm{M} \text { (colorimetric method) } \\
0.15 \mu \mathrm{M} \text { (fluorescence method) }\end{array}$ & Su et al. (2020) \\
\hline- & - & $\begin{array}{l}1.67 \mu \mathrm{M}- \\
2.01 \mathrm{mM}\end{array}$ & $0.35 \mu \mathrm{M}$ & Yang et al. (2021b) \\
\hline- & Glucose & - & $\begin{array}{c}0.15 \mu \mathrm{M} \text { (colorimetric method fluorescence } \\
\text { method) }\end{array}$ & Su et al. (2020) \\
\hline- & Xanthine & - & $\begin{array}{c}0.11 \mu \mathrm{M} \text { (colorimetric method) } \\
0.12 \mu \mathrm{M} \text { (fluorescence method) }\end{array}$ & Su et al. (2020) \\
\hline- & Hydroquinone $\left(\mathrm{H}_{2} \mathrm{Q}\right)$ & $\begin{array}{l}0.05-20 \mathrm{mM} \\
1-30 \mathrm{mM}\end{array}$ & $1 \mu \mathrm{M}$ & Ren et al. (2015) \\
\hline 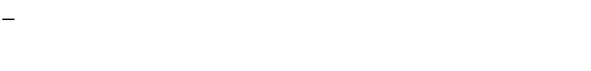 & Cholesterol & $\begin{array}{l}1.66 \mu \mathrm{M}- \\
1.65 \mathrm{mM}\end{array}$ & $0.49 \mu \mathrm{M}$ & Yang et al. (2021b) \\
\hline $\begin{array}{l}\text { Collaborative detection by colorimetric and SERS } \\
\text { methods }\end{array}$ & Uric acid & - & $\begin{array}{c}1-500 \mu \mathrm{M} \text { (colorimetric method) } \\
0.01-500 \mu \mathrm{M} \\
\text { (SERS method) }\end{array}$ & Wang et al. (2019) \\
\hline- & Glucose & - & $5 \times 10^{-7} \mathrm{M}$ & Gan et al. (2021) \\
\hline Double emission carbon spot detection & O-phenylenediamine & - & $0.58 \mu \mathrm{M}$ & $\begin{array}{l}\text { Mathivanan et al. } \\
\text { (2020) }\end{array}$ \\
\hline- & $\mathrm{H}_{2} \mathrm{O}_{2}$ & - & $0.27 \mu \mathrm{M}$ & $\begin{array}{l}\text { Mathivanan et al. } \\
(2020)\end{array}$ \\
\hline- & Hydroquinone $\left(\mathrm{H}_{2} \mathrm{Q}\right)$ & $1.0-75 \mu \mathrm{M}$ & $0.04 \mu \mathrm{M}$ & Wang et al. (2019) \\
\hline
\end{tabular}

simulated using finite-difference time-domain (FDTD) method to demonstrate how the aggregation of gold NPs enhanced the electromagnetic field, thereby increasing SERS signal based on diamond-like nanocomposite. The nanocomposite enables glucose detection at a concentration of $5 \times 10^{-7} \mathrm{M}$ (Gan et al., 2021). All these demonstrated that the synergistic method based on colorimetric reaction and SERS detection possessed the advantages of a low detection limit, a wide detection range, and high accuracy, which made the detection results more reliable and accurate.

\section{Double Emission Carbon Spot Detection}

Using a two-carbon point system as a peroxide-mimicking enzyme and a fluorescent probe, combining carbon point with catalytic activity or carbon point with fluorescence quenching effect greatly improves the sensitivity of the detection method.

Dhamodiran Mathivanan et al. synthesized double emission carbon spots of enzyme simulated N/Cl-CDs and N/Zn-CDs. $\mathrm{N} / \mathrm{Cl}-\mathrm{CDs}$ exhibited apparent intrinsic peroxidase-like activity, catalyzing OPD oxidation by $\mathrm{H}_{2} \mathrm{O}_{2}$ to form the yellow product 2, 3-diaminophenazine. N/Zn-CDs exhibited significant fluorescence properties, with a quantum yield of 27.52\% (Mathivanan et al., 2020). Using similar construction, Wang et al. constructed a double-carbon point system with fluorescent CDs (N/Cl-CDs) and copper-doped $\mathrm{CDs}(\mathrm{N} / \mathrm{Cu}-\mathrm{CDs})$ that function as peroxide mimic and fluorescent probe and can fluoresce in hydroquinone determination. The fluorescence quantum yield of N/Cu-CDs was 37\%. Compared with the study of Dhamodiran Mathivanan et al., the fluorescence quantum yield was significantly improved. N/Cl-CDs exhibits inherent peroxidase-like activity and catalyzes hydroquinone oxidation to p-benzoquinone and intermediates to determine $\mathrm{H}_{2} \mathrm{Q}$ (Wang $\mathrm{X}$. et al., 2020).

Although nanozymes with complex CDs have the advantages of rapid response, high sensitivity, and simplicity when applied to molecular detection, they possess some limitations and are unsuitable for in vivo and continuous analyses. However, they lay the foundation for enzyme-dependent biological research. In future studies, it is necessary to enhance the substrate specificity of CDs complex nanozymes by modifying their functional groups.

To clearly describe the application performance of CDs-based nanozymes in the field of detection, we summarized the existing reports in Table 1 .

\section{DISSCUSSION}

In the past 10 years, CDs-based nanozymes have progressed in expanding the types of nanozymes, understanding the reaction mechanism, and regulating their catalytic performance, but numerous problems remain.

1) There is limited information on the biological characteristics of CDs-based nanozymes in vivo. The biological effects of 
CDs-based nanozymes should be systematically described, including their cytotoxicity, in vivo properties, biological distribution, and pharmacokinetics to facilitate their broad applications in cancer treatment, ROS removal, and inflammation alleviation.

2) The detailed system mechanism of CDs-based nanozymes remains unclear, and the relationship between the catalytic mechanism and its structure requires further investigation. By studying their structures, it is feasible to integrate enzyme-like activities and catalytic mechanisms of various nanozymes. In addition, a well-defined coordination structure can provide a clear experimental model for studying the underlying mechanism, and computational simulation can better design nanozymes with CDs.

3) To date, most CDs-based nanozymes exhibit only oxidoreductaselike activity. Given the numerous enzyme-catalyzed biochemical reactions in nature, it is necessary to further develop novel CDsbased nanozymes with a wider range of enzyme activities. In addition to stimulating proteases, it may be a breakthrough direction to broaden the simulation objects of nucleic acid-

\section{REFERENCES}

Asati, A., Santra, S., Kaittanis, C., Nath, S., and Perez, J. M. (2009). Oxidase-Like Activity of Polymer-Coated Cerium Oxide Nanoparticles. Angew. Chem. Int. Edition 48 (13), 2308-2312. doi:10.1002/anie.200805279

Attar, F., Shahpar, M. G., Rasti, B., Sharifi, M., Saboury, A. A., Rezayat, S. M., et al. (2019). Nanozymes with Intrinsic Peroxidase-like Activities. J. Mol. Liquids. 278, 130-144. doi:10.1016/j.molliq.2018.12.011

Chen, C.-Y., Tan, Y. Z., Hsieh, P.-H., Wang, C.-M., Shibata, H., Maejima, K., et al. (2020b). Metal-Free Colorimetric Detection of Pyrophosphate Ions by Inhibitive Nanozymatic Carbon Dots. ACS Sens. 5 (5), 1314-1324. doi:10.1021/acssensors.9b02486

Chen, K., Chou, W., Liu, L., Cui, Y., Xue, P., and Jia, M. (2019). Electrochemical Sensors Fabricated by Electrospinning Technology: An Overview. Sensors 19, 3676. doi:10.3390/s19173676

Chen, Q., Li, S., Liu, Y., Zhang, X., Tang, Y., Chai, H., et al. (2020a). Sizecontrollable Fe-N/C Single-Atom Nanozyme with Exceptional Oxidase-like Activity for Sensitive Detection of Alkaline Phosphatase. Sensors Actuators B: Chem. 305, 127511. doi:10.1016/j.snb.2019.127511

Cheng, H., Zhang, L., He, J., Guo, W., Zhou, Z., Zhang, X., et al. (2016). Integrated Nanozymes with Nanoscale Proximity for In Vivo Neurochemical Monitoring in Living Brains. Anal. Chem. 88 (10), 5489-5497. doi:10.1021/ acs.analchem.6b00975

Ding, H., Hu, B., Zhang, B., Zhang, H., Yan, X., Nie, G., et al. (2020). Carbon-based Nanozymes for Biomedical Applications. Nano Res. 14 (3), 570-583. doi:10.1007/s12274-020-3053-9

Ding, Y., Liu, H., Gao, L.-N., Fu, M., Luo, X., Zhang, X., et al. (2019). Fe-doped Ag2S with Excellent Peroxidase-like Activity for Colorimetric Determination of H2O2. J. Alloys Comp. 785, 1189-1197. doi:10.1016/j.jallcom.2019.01.225

Du, J., Qi, S., Chen, J., Yang, Y., Fan, T., Zhang, P., et al. (2020). Fabrication of Highly Active Phosphatase-like Fluorescent Cerium-Doped Carbon Dots for In Situ Monitoring the Hydrolysis of Phosphate Diesters. RSC Adv. 10 (68), 41551-41559. doi:10.1039/d0ra07429b

Fan, K., Xi, J., Fan, L., Wang, P., Zhu, C., Tang, Y., et al. (2018). In Vivo guiding Nitrogen-Doped Carbon Nanozyme for Tumor Catalytic Therapy. Nat. Commun. 9, 1. doi:10.1038/s41467-018-03903-8

Fasciani, C., Silvero, M. J., Anghel, M. A., Argüello, G. A., Becerra, M. C., and Scaiano, J. C. (2014). Aspartame-Stabilized Gold-Silver Bimetallic Biocompatible Nanostructures with Plasmonic Photothermal Properties, based enzymes, such as graphene oxide, as a photocatalytic nuclease, which could cleave DNA.

\section{AUTHOR CONTRIBUTIONS}

JJ drafted the manuscript. KS, SX, ZL, and LZ guided and amended the manuscript. SX and KS helped to review the manuscript. All authors contributed to the manuscript.

\section{FUNDING}

The authors appreciate the financial supports by the National Natural Science Foundation of China (31870486), (31600364), the Natural Science Foundation of Jilin Province (YDZJ202101ZYTS092), the Science and Technology Project of Jilin Provincial Department of Education (JJKH20181170KJ), the Natural Science Foundation of Changchun Normal University 2019 (010), 2019 (018), KXK (2020) 002.

Antibacterial Activity, and Long-Term Stability. J. Am. Chem. Soc. 136 (50), 17394-17397. doi:10.1021/ja510435u

Fischbach, M. A., and Walsh, C. T. (2009). Antibiotics for Emerging Pathogens. Science 325 (5944), 1089-1093. doi:10.1126/science.1176667

Gan, H., Han, W., Fu, Z., and Wang, L. (2021). The Chain-like Au/carbon Dots Nanocomposites with Peroxidase-like Activity and Their Application for Glucose Detection. Colloids Surf. B: Biointerfaces. 199, 111553. doi:10.1016/ j.colsurfb.2020.111553

Gao, L., Zhuang, J., Nie, L., Zhang, J., Zhang, Y., Gu, N., et al. (2007). Intrinsic Peroxidase-like Activity of Ferromagnetic Nanoparticles. Nat. Nanotech. 2 (9), 577-583. doi:10.1038/nnano.2007.260

Guo, J., Wang, Y., and Zhao, M. (2019). Target-directed Functionalized Ferrous Phosphate-Carbon Dots Fluorescent Nanostructures as Peroxidase Mimetics for Cancer Cell Detection and ROS-Mediated Therapy. Sensors Actuators B: Chem. 297, 126739. doi:10.1016/j.snb.2019.126739

Hermanová, S., and Pumera, M. (2020). Biocatalytic Micro- and Nanomotors. Chem. Eur. J. 26 (49), 11085-11092. doi:10.1002/chem.202001244

Honarasa, F., Kamshoori, F. H., Fathi, S., and Motamedifar, Z. (2019). Carbon Dots on V2O5 Nanowires Are a Viable Peroxidase Mimic for Colorimetric Determination of Hydrogen Peroxide and Glucose. Microchim Acta. 186 (4), 234. doi:10.1007/s00604-019-3344-6

Honarasa, F., Keshtkar, S., Eskandari, N., and Eghbal, M. (2021). Catalytic and Electrocatalytic Activities of Fe3O4/CeO2/C-Dot Nanocomposite. Chem. Pap. 75 (6), 2371-2378. doi:10.1007/s11696-020-01443-4

Hou, S., Hu, X., Wen, T., Liu, W., and Wu, X. (2013). Core-Shell Noble Metal Nanostructures Templated by Gold Nanorods. Adv. Mater. 25 (28), 3857-3862. doi:10.1002/adma.201301169

$\mathrm{Hu}, \mathrm{S}$., Zhang, W., Li, N., Chang, Q., and Yang, J. (2021). Integrating Biphase $\gamma$ - and a-Fe2O3 with Carbon Dots as a Synergistic Nanozyme with Easy Recycle and High Catalytic Activity. Appl. Surf. Sci. 545, 148987. doi:10.1016/j.apsusc.2021.148987

Jin, Z., Xu, G., Niu, Y., Ding, X., Han, Y., Kong, W., et al. (2020). Ti3C2Tx MXeneDerived TiO2/C-QDs as Oxidase Mimics for the Efficient Diagnosis of Glutathione in Human Serum. J. Mater. Chem. B. 8 (16), 3513-3518. doi:10.1039/c9tb02478f

Kang, Z., and Lee, S.-T. (2019). Carbon Dots: Advances in Nanocarbon Applications. Nanoscale 11 (41), 19214-19224. doi:10.1039/c9nr05647e

Kluenker, M., Nawaz Tahir, M., Ragg, R., Korschelt, K., Simon, P., Gorelik, T. E., et al. (2017).Pd@Fe2O3 Superparticles with Enhanced Peroxidase Activity by Solution Phase Epitaxial Growth. Chem. Mater. 29 (3), 1134-1146. doi:10.1021/ acs.chemmater.6b04283 
Kohanski, M. A., Dwyer, D. J., and Collins, J. J. (2010). How Antibiotics Kill Bacteria: from Targets to Networks. Nat. Rev. Microbiol. 8 (6), 423-435. doi:10.1038/nrmicro2333

Leidinger, P., Treptow, J., Hagens, K., Eich, J., Zehethofer, N., Schwudke, D., et al. (2015). Isoniazid@Fe2O3Nanocontainers and Their Antibacterial Effect on Tuberculosis Mycobacteria. Angew. Chem. Int. Ed. 54 (43), 12597-12601. doi:10.1002/anie.201505493

Li, B., Chen, D., Nie, M., Wang, J., Li, Y., and Yang, Y. (2018). Carbon Dots/Cu2 O Composite with Intrinsic High Protease-like Activity for Hydrolysis of Proteins under Physiological Conditions. Part. Part. Syst. Charact. 35, 1800277. doi:10.1002/ppsc.201800277

Li, F., Chang, Q., Li, N., Xue, C., Liu, H., Yang, J., et al. (2020a). Carbon DotsStabilized Cu4O3 for a Multi-Responsive Nanozyme with Exceptionally High Activity. Chem. Eng. J. 394, 125045. doi:10.1016/j.cej.2020.125045

Li, F., Li, N., Xue, C., Wang, H., Chang, Q., Liu, H., et al. (2020b). A Cu2O-CDs-Cu Three Component Catalyst for Boosting Oxidase-like Activity with Hot Electrons. Chem. Eng. J. 382, 122484. doi:10.1016/j.cej.2019.122484

Li, Y.-F., Lin, Z.-Z., Hong, C.-Y., and Huang, Z.-Y. (2021). Histamine Detection in Fish Samples Based on Indirect Competitive ELISA Method Using Iron-Cobalt Co-doped Carbon Dots Labeled Histamine Antibody. Food Chem. 345, 128812. doi:10.1016/j.foodchem.2020.128812

Liang, C., Lan, Y., Sun, Z., Zhou, L., Li, Y., Liang, X., et al. (2020). Synthesis of Carbon Quantum Dots with Iron and Nitrogen from Passiflora edulis and Their Peroxidase-Mimicking Activity for Colorimetric Determination of Uric Acid. Microchim Acta. 187 (7), 405. doi:10.1007/s00604-020-04391-8

Lin, Y., Ren, J., and Qu, X. (2014). Catalytically Active Nanomaterials: A Promising Candidate for Artificial Enzymes. Acc. Chem. Res. 47 (4), 1097-1105. doi:10.1021/ar400250z

Liu, H., Ding, Y.-N., Yang, B., Liu, Z., Zhang, X., and Liu, Q. (2018). Iron Doped $\mathrm{CuSn}(\mathrm{OH}) 6$ Microspheres as a Peroxidase-Mimicking Artificial Enzyme for H2O2 Colorimetric Detection. ACS Sustain. Chem. Eng. 6 (11), 14383-14393. doi:10.1021/acssuschemeng.8b03082

Lu, C., Liu, Y., Wen, Q., Liu, Y., Wang, Y., Rao, H., et al. (2020). Ratiometric Fluorescence Assay for L-Cysteine Based on Fe-Doped Carbon Dot Nanozymes. Nanotechnology 31, 445703. doi:10.1088/1361-6528/aba578

Luo, N., Yang, Z., Tang, F., Wang, D., Feng, M., Liao, X., et al. (2019). Fe3O4/Carbon Nanodot Hybrid Nanoparticles for the Indirect Colorimetric Detection of Glutathione. ACS Appl. Nano Mater. 2 (6), 3951-3959. doi:10.1021/acsanm.9b00854

Ma, X., Hortelão, A. C., Patiño, T., and Sánchez, S. (2016). Enzyme Catalysis to Power Micro/Nanomachines. ACS Nano 10 (10), 9111-9122. doi:10.1021/ acsnano.6b04108

Mathesh, M., Sun, J., and Wilson, D. A. (2020). Enzyme Catalysis Powered Micro/ nanomotors for Biomedical Applications. J. Mater. Chem. B. 8 (33), 7319-7334. doi:10.1039/d0tb01245a

Mathivanan, D., Tammina, S. K., Wang, X., and Yang, Y. (2020). Dual Emission Carbon Dots as Enzyme Mimics and Fluorescent Probes for the Determination of O-Phenylenediamine and Hydrogen Peroxide. Microchim Acta. 187 (5), 292. doi:10.1007/s00604-020-04256-0

Natalio, F., André, R., Hartog, A. F., Stoll, B., Jochum, K. P., Wever, R., et al. (2012). Vanadium Pentoxide Nanoparticles Mimic Vanadium Haloperoxidases and Thwart Biofilm Formation. Nat. Nanotech. 7 (8), 530-535. doi:10.1038/ nnano.2012.91

Nirala, N. R., Pandey, S., Bansal, A., Singh, V. K., Mukherjee, B., Saxena, P. S., et al. (2015). Different Shades of Cholesterol: Gold Nanoparticles Supported on MoS2 Nanoribbons for Enhanced Colorimetric Sensing of Free Cholesterol. Biosens. Bioelectron. 74, 207-213. doi:10.1016/j.bios.2015.06.043

Qin, X., Dong, Y., Wang, M., Zhu, Z., Li, M., Chen, X., et al. (2018). C-dots Assisted Synthesis of Gold Nanoparticles as Labels to Catalyze Copper Deposition for Ultrasensitive Electrochemical Sensing of Proteins. Sci. China Chem. 61 (4), 476-482. doi:10.1007/s11426-017-9204-8

Ren, X., Liu, J., Ren, J., Tang, F., and Meng, X. (2015). One-pot Synthesis of Active Copper-Containing Carbon Dots with Laccase-like Activities. Nanoscale 7 (46), 19641-19646. doi:10.1039/c5nr04685h

Rizzello, L., and Pompa, P. P. (2014). Nanosilver-based Antibacterial Drugs and Devices: Mechanisms, Methodological Drawbacks, and Guidelines. Chem. Soc. Rev. 43 (5), 1501-1518. doi:10.1039/c3cs60218d

Shu, X., Chang, Y., Wen, H., Yao, X., and Wang, Y. (2020). Colorimetric Determination of Ascorbic Acid Based on Carbon Quantum Dots as
Peroxidase Mimetic Enzyme. RSC Adv. 10 (25), 14953-14957. doi:10.1039/ d0ra02105a

Song, Y.-Y., Yang, T., Cao, J., Gao, Z., and Lynch, R. P. (2012). Protein-mediated Synthesis of Antibacterial Silver Nanoparticles Deposited on Titanium Dioxide Nanotube Arrays. Microchim Acta. 177 (1-2), 129-135. doi:10.1007/s00604012-0769-6

Su, L., Cai, Y., Wang, L., Dong, W., Mao, G., Li, Y., et al. (2020). Hemin@carbon Dot Hybrid Nanozymes with Peroxidase Mimicking Properties for Dual (Colorimetric and Fluorometric) Sensing of Hydrogen Peroxide, Glucose and Xanthine. Microchim Acta. 187 (2), 132. doi:10.1007/s00604-019-4103-4

Tan, X., Zhang, L., Tang, Q., Zheng, G., and Li, H. (2019). Ratiometric Fluorescent Immunoassay for the Cardiac Troponin-I Using Carbon Dots and PalladiumIridium Nanocubes with Peroxidase-Mimicking Activity. Microchim Acta. 186 (5), 280. doi:10.1007/s00604-019-3375-z

Teymourian, H., Parrilla, M., Sempionatto, J. R., Montiel, N. F., Barfidokht, A., Van Echelpoel, R., et al. (2020). Wearable Electrochemical Sensors for the Monitoring and Screening of Drugs. ACS Sens. 5 (9), 2679-2700. doi:10.1021/acssensors.0c01318

Tripathi, K. M., Ahn, H. T., Chung, M., Le, X. A., Saini, D., Bhati, A., et al. (2020). N, S, and P-Co-Doped Carbon Quantum Dots: Intrinsic Peroxidase Activity in a Wide pH Range and its Antibacterial Applications. ACS Biomater. Sci. Eng. 6 (10), 5527-5537. doi:10.1021/acsbiomaterials.0c00831

Vinita, Nirala, N. R., and Prakash, R. (2018). One Step Synthesis of AuNPs@MoS 2 -QDs Composite as a Robust Peroxidase- Mimetic for Instant Unaided Eye Detection of Glucose in Serum, Saliva and Tear. Sensors Actuators B: Chem. 263, 109-119. doi:10.1016/j.snb.2018.02.085

Wang, A., Guan, C., Shan, G., Chen, Y., Wang, C., and Liu, Y. (2019). A Nanocomposite Prepared from Silver Nanoparticles and Carbon Dots with Peroxidase Mimicking Activity for Colorimetric and SERS-Based Determination of Uric Acid. Microchim Acta. 186 (9), 644. doi:10.1007/ s00604-019-3759-0

Wang, J., Zhu, Y., and Wang, L. (2019). Synthesis and Applications of RedEmissive Carbon Dots. Chem. Rec. 19 (10), 2083-2094. doi:10.1002/ tcr.201800172

Wang, Q., Xue, Q., Chen, T., Li, J., Liu, Y., Shan, X., et al. (2021b). Recent Advances in Electrochemical Sensors for Antibiotics and Their Applications. Chin. Chem. Lett. 32 (2), 609-619. doi:10.1016/j.cclet.2020.10.025

Wang, X., Cheng, Z., Zhou, Y., Tammina, S. K., and Yang, Y. (2020b). A Double Carbon Dot System Composed of N, Cl-Doped Carbon Dots and N, Cu-Doped Carbon Dots as Peroxidase Mimics and as Fluorescent Probes for the Determination of Hydroquinone by Fluorescence. Microchim Acta. 187 (6), 350. doi:10.1007/s00604-020-04322-7

Wang, X., Lu, Y., Hua, K., Yang, D., and Yang, Y. (2021a). Iodine-doped Carbon Dots with Inherent Peroxidase Catalytic Activity for Photocatalytic Antibacterial and Wound Disinfection. Anal. Bioanal. Chem. 413 (5), 1373-1382. doi:10.1007/s00216-020-03100-X

Wang, Y., Wang, Z., Rui, Y., and Li, M. (2015). Horseradish Peroxidase Immobilization on Carbon nanodots/CoFe Layered Double Hydroxides: Direct Electrochemistry and Hydrogen Peroxide Sensing. Biosens. Bioelectron. 64, 57-62. doi:10.1016/j.bios.2014.08.054

Wang, Z., Zhang, R., Yan, X., and Fan, K. (2020a). Structure and Activity of Nanozymes: Inspirations for De Novo Design of Nanozymes. Mater. Today. 41, 81-119. doi:10.1016/j.mattod.2020.08.020

Wei, H., and Wang, E. (2013). Nanomaterials with Enzyme-like Characteristics (Nanozymes): Next-Generation Artificial Enzymes. Chem. Soc. Rev. 42, 6060. doi: $10.1039 / \mathrm{c} 3 \operatorname{cs} 35486 \mathrm{e}$

Woo, M.-A., Kim, M., Jung, J., Park, K., Seo, T., and Park, H. (2013). A Novel Colorimetric Immunoassay Utilizing the Peroxidase Mimicking Activity of Magnetic Nanoparticles. Ijms 14 (5), 9999-10014. doi:10.3390/ ijms 14059999

Xu, D., Zhan, C., Sun, Y., Dong, Z., Wang, G. P., and Ma, X. (2019). Turn-NumberDependent Motion Behavior of Catalytic Helical Carbon Micro/Nanomotors. Chem. Asian J. 14 (14), 2497-2502. doi:10.1002/asia.201900386

Yang, C., Aslan, H., Zhang, P., Zhu, S., Xiao, Y., and Chen, L. (2020). Carbon Dotsfed Shewanella Oneidensis MR-1 For Bioelectricity Enhancement. Nat. Commun. 11, 1379. doi:10.1038/s41467-020-14866-0

Yang, L., Liu, X., Lu, Q., Huang, N., Liu, M., Zhang, Y., et al. (2016). Catalytic and Peroxidase-like Activity of Carbon Based-AuPd Bimetallic Nanocomposite 
Produced Using Carbon Dots as the Reductant. Analytica Chim. Acta. 930, 23-30. doi:10.1016/j.aca.2016.04.041

Yang, Q., Gao, Y., Xu, L., Hong, W., She, Y., and Yang, G. (2021a). Enzyme-driven Micro/nanomotors: Recent Advances and Biomedical Applications. Int. J. Biol. Macromolecules. 167, 457-469. doi:10.1016/j.ijbiomac.2020.11.215

Yang, W., Huang, T., Zhao, M., Luo, F., Weng, W., Wei, Q., et al. (2017). High Peroxidaselike Activity of Iron and Nitrogen Co-doped Carbon Dots and its Application in Immunosorbent Assay. Talanta 164, 1-6. doi:10.1016/j.talanta.2016.10.099

Yang, Z., Liu, Y., Lu, C., Yue, G., Wang, Y., Rao, H., et al. (2021b). One-pot Synthesis of CeO2-Carbon Dots with Enhanced Peroxidase-like Activity and Carbon Dots for Ratiometric Fluorescence Detection of $\mathrm{H} 2 \mathrm{O} 2$ and Cholesterol. J. Alloys Comp. 862, 158323. doi:10.1016/j.jallcom.2020.158323

Yuan, H., Liu, X., Wang, L., and Ma, X. (2021). Fundamentals and Applications of Enzyme Powered Micro/nano-Motors. Bioactive Mater. 6 (6), 1727-1749. doi:10.1016/j.bioactmat.2020.11.022

Zhan, Y., Yang, S., Luo, F., Guo, L., Zeng, Y., Qiu, B., et al. (2020). Emission Wavelength Switchable Carbon Dots Combined with Biomimetic Inorganic Nanozymes for a Two-Photon Fluorescence Immunoassay. ACS Appl. Mater. Inter. 12 (27), 30085-30094. doi:10.1021/acsami.0c06240

Zhang, J., Lu, X., Tang, D., Wu, S., Hou, X., Liu, J., et al. (2018). Phosphorescent Carbon Dots for Highly Efficient Oxygen Photosensitization and as PhotoOxidative Nanozymes. ACS Appl. Mater. Inter. 10 (47), 40808-40814. doi:10.1021/acsami.8b15318

Zhao, L., Ren, X., Zhang, J., Zhang, W., Chen, X., and Meng, X. (2020). Dendritic Silica with Carbon Dots and Gold Nanoclusters for Dual Nanozymes. New J. Chem. 44 (5), 1988-1992. doi:10.1039/c9nj05655f
Zhao, L., Wu, Z., Liu, G., Lu, H., Gao, Y., Liu, F., et al. (2019). High-activity Mo, S Co-doped Carbon Quantum Dot Nanozyme-Based cascade Colorimetric Biosensor for Sensitive Detection of Cholesterol. J. Mater. Chem. B. 7 (44), 7042-7051. doi:10.1039/c9tb01731c

Zhuo, S., Fang, J., Li, M., Wang, J., Zhu, C., and Du, J. (2019). Manganese(II)-doped Carbon Dots as Effective Oxidase Mimics for Sensitive Colorimetric Determination of Ascorbic Acid. Microchim Acta. 186 (12), 745. doi:10.1007/s00604-019-3887-6

Conflict of Interest: The authors declare that the research was conducted in the absence of any commercial or financial relationships that could be construed as a potential conflict of interest.

Publisher's Note: All claims expressed in this article are solely those of the authors and do not necessarily represent those of their affiliated organizations, or those of the publisher, the editors and the reviewers. Any product that may be evaluated in this article, or claim that may be made by its manufacturer, is not guaranteed or endorsed by the publisher.

Copyright $\odot 2021 \mathrm{Jin}, \mathrm{Li}$, Zhang, Luan, Xin and Song. This is an open-access article distributed under the terms of the Creative Commons Attribution License (CC BY). The use, distribution or reproduction in other forums is permitted, provided the original author(s) and the copyright owner(s) are credited and that the original publication in this journal is cited, in accordance with accepted academic practice. No use, distribution or reproduction is permitted which does not comply with these terms. 